\title{
A Quick Study of a Single Image Defogging Algorithm
}

\author{
Yanhai Wu ${ }^{1, a}$, Jing Zhang ${ }^{1, b}$,Kang Chen ${ }^{1}$, Lihua Pang ${ }^{1}$ \\ ${ }^{1}$ College of Communication and Information Engineering, Xi'an University of Science and \\ Technology, Xi'an ,710054,China \\ aemail:wyh7388@163.com, bemail:18700186109@163.com
}

\begin{abstract}
Keywords: image defogging; mean filter; guide filter; atmospheric dissipation function
\end{abstract}
\begin{abstract}
In foggy conditions, acquired images have low contrast ratio and low scene visibility problems. The traditional defogging algorithm has high time complexity and low speed characteristics and can not be applied in real-time image processing. Thus, a single image restoration method based on mean filter is proposed in this paper combined with the optical properties of atmospheric. In this method, on the basis of atmospheric scattering model, accurate atmospheric dissipation function can be achieved firstly by using mean filter. Atmosphere light is obtained by quadtree algorithm which is more efficient. Finally, restored image is calculated by the atmospheric scattering model and the brightness of the image is adjusted, and a clear image without fog is gained. Simulation results show that this algorithm has a strong adaptability to various scenes and restored images have plentiful colors. Compared with the classic defogging algorithm, this algorithm ensures the defogging effect while overcomes the high time complexity and low speed defects of guide filter algorithm.
\end{abstract}

\section{Introduction}

Fog and haze suspended in the atmosphere with a large number of tiny particles make light spread with the absorption and scattering. Thus, the obtained image has decreased image contrast and color distortion, which seriously affect the road monitoring system of information extraction and bring serious security risks. Therefore, effective and fast defogging method has important application value.

Current, image dehazing is mainly divided into two categories, one is based on fog image enhancement. The common methods are histogram equalization, curvelet transform, Retinex algorithm etc. The common characteristic is without considering the degradation reasons, only from the visual to improve image clarity and increase the image details information. Another is based on the image restoration of atmospheric scattering model, using a priori information, inverse deduction operation, to obtain the optimal estimate of the image without degradation of the image, and then restore the fog degraded image. In recent years, single image dehazing with its easy obtained parameters, less auxiliary information, low cost and good defogging effect has been favored by many scholars. The main principle is using the image itself structural constraints on scene albedo assumption of rate or the depth of the scene. Atmospheric scattering model parameters are estimated from a single image to fog. He et al. [1] based on statistics of no fog image, proposes a defogging method based on dark channel prior. Firstly using minimum coarse filtering to estimate transmissivity. Matting algorithm was then used to refine the transmittance, and restoration of fog degraded image. But the algorithm has image edge fog prone residual mist, and the time complexity of the algorithm is high and cannot meet the requirements of real-time processing. Tarel et al. [2], based on filtering and preprocessing idea, assumed that the atmospheric scattering function in the feasible region close to the maximum value, the use of the median filter to estimate the form, and then restore the fog image. The method is fast, but the median filter does not have the edge preserving property, and the whole is dark, the image of the scene is complex, and the recovery effect is poor. Yu et al [3] proposed a fast automatic defogging algorithm, first conducting atmospheric light white balance, then the bilateral filtering with the edge preserving is used to estimate atmospheric dissipation function, seeking a simplified atmospheric scattering model for 
image restoration, the algorithm processing speed is fast, but the white object recovery effect is not good.

In view of the shortcomings of the current algorithm, the clear and foggy images are recovered from the following 3 aspects. 1)the mean filter is used to estimate the atmospheric dissipation function, which can effectively avoid the phenomenon of residual fog and local; 2) in order to avoid the impact of the A value of the bright object in the fog, the method of the quadtree method is used to estimate the A value of atmospheric light intensity accurately and quickly; 3) to adjust the brightness of the restoration results, in order to achieve a better visual effect. Experimental results show that the proposed algorithm can overcome the shortcomings of traditional algorithm with high time complexity and slow speed.

\section{Physical model of atmospheric degradation}

In machine vision, the fog image restoration technique is based on the atmospheric degradation physical model proposed by [4] et al. Narasimhan. The model describes the process of image degradation, which can be expressed as

$I(x)=J(x) t(x)+A(1-t(x))$

Among them, $I(x)$ observed for foggy images; $J(x)$ for the same scene albedo, that is, to restore the no fog image; $A$ is atmospheric light intensity at infinity; $t(x)$ presents rate along the light propagation, reflects the light to penetrate the fog. From formula (1) it can be seen that the atmospheric scattering model consists of two parts: direct attenuation and atmospheric dissipation function, In order to facilitate the atmospheric dissipation function is expressed as

$$
V(x)=A(1-t(x))
$$

The resulting $\mathrm{V}(\mathrm{x})=\mathrm{A}(1-\mathrm{t}(\mathrm{x}))$ into equation (1)

$$
J(x)=\frac{A(I(x)-V(x))}{A-V(x)}
$$

From equation (3) dissipation function $V(x)$ and global atmospheric light $A$ quickly and accurately is estimated from the fog image $I(x)$, recovering the fog image clear $J(x)$.

\section{A fast image dehazing algorithm}

\subsection{Based on mean filter $V(x)$ estimated}

From atmospheric degradation of the physical model, atmospheric light scattering function should satisfy two constraints: first, atmospheric dissipation function argument should be positive, that is, each pixel point $V(x) \geq 0$; second, the atmosphere atomizing dissipation function is not greater than the image $I(x)$ is the minimum color component $M(x)$, i.e., $V(x) \leq M(x)$, where $M(x)$ can be expressed as

$$
M(x)=\sum_{c \in\{R, G, B\}}\left(I^{c}(x)\right)
$$

Where $I^{c}(x)$ indicates an image pixel values c channel at the point $\mathrm{x}$, c represents the primary colors, it is advisable $\mathrm{G}, \mathrm{R}, \mathrm{B}$ in a channel value. $M(x)$ contains the image edge features and rich texture, its brightness value does not accurately represent the fog density, and therefore need to further eliminate the influence of $M(x)$ unnecessary texture detail information. Substituted into the formula (2) can be obtained

$$
t(x) \geq 1-\frac{M(x)}{A}
$$

In this paper, the mean filter is used to estimate the transmittance of $t(x)$, such as (6). The $\Omega(x)$ represents the $S \times S$ neighborhood of the pixel point $\mathrm{S}$, and the $x$ is the size of the mean filter window. 


$$
\text { average }_{s}\left(1-\frac{M(x)}{A}\right)=1-\frac{\text { average }_{s}(M(x))}{A}=1-\frac{\sum_{y \in \Omega(x)} M(y)}{A S^{2}}
$$

But the image mean filtering does not accurately reflect $t(x)$, just with the $t(x)$ keeping the same changing trend, so for mean filtering results there were some compensation, and remember to $M_{\text {ave }}(x)=$ average $_{s}(M(x))$. Hence, we obtain transmissivity values for:

$$
t(x)=1-\frac{M_{a v e}(x)}{A}+\phi \frac{M_{a v e}(x)}{A}=1-\sigma \frac{M_{a v e}(x)}{A}
$$

Among them, $\sigma=1-\phi$, when the $\sigma$ value is larger, the transmittance is small, no fog image restoration is dark, and partial light; in order to make the restoration effect more stable, adapting to different scenes, the introduction of adjusting parameter $e$, and the $e=0.2$.To $\sigma=e m_{a v}$, where $M(x)$ is the average of all the pixels in the $m_{a v}$, and with the overall gray level of the image of the fog, that is, the role of adaptive adjustment of brightness. In order to ensure that the results of the mean filtering is compensated, $\phi$ is positive and can not be too small, this paper takes the upper limit of $\phi$ is 0.9 , so we can get $\sigma=\min \left(e m_{a v}, 0.9\right)$. To sum up:

$$
V(x)=\min \left(\min \left(e m_{a v}, \rho\right) M_{a v e}(x), M(x)\right)
$$

In Figure 1, after obtaining the atmospheric dissipation function, we obtain the minimum color channel (b) from the fog image (a), and then get the image of the atmospheric scattering function (c) by means of the mean filter.
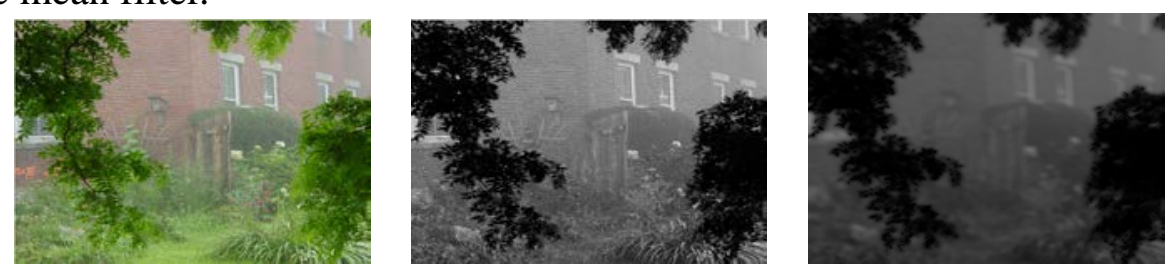

(a) Foggy image (b) Minimum path graph (c) Atmospheric dissipation function Fig.1. Atmospheric dissipation function estimation

\subsection{A value-based quadtree segmentation estimate}

Estimation of atmospheric light fog often choose the pixel image in fog drop and the largest concentration of value as the atmospheric light intensity of $A$. But the actual scene is often more complex, especially when the bright region or white objects exists, estimation of atmospheric light will have a large deviation. Later, He et al. [1] select the $0.1 \%$ pixels of the brightest pixels in the dark passage, and then select the most bright pixels in the original atomization image as the estimation of atmospheric light intensity $A$.This method can be more accurate to estimate the value of atmospheric light, but for bright white building scenes or images it generated errors and the speed is slow; so in order to quickly and accurately estimate the value of atmospheric light, we use the quad-tree subdivision scheme [5] to improve it. First find the minimum value of the image of the channel, in order to avoid local image on a channel exists great value caused by the error estimation; then channel minimum image is divided into four regions, we calculated the regional average brightness, take regional mean maximum repeat region division, straight to the size of the area is less than the given threshold, generally set the threshold value of $20 \times 20$;Finally, calculate the atmospheric fog light original figure R, G, B channel in this block mean $\bar{R}, \bar{G}, \bar{B}$, the resulting image according to the position marked $A$. The method converges fast, positions accurately and reduce the time complexity. Using quadtree segmentation method to estimate atmospheric light of the process shown in Figure 2. 

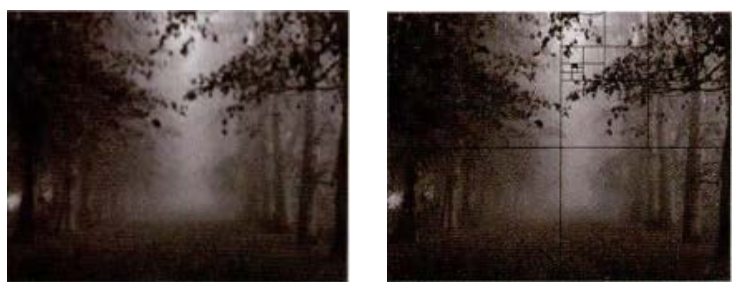

(a) Minimum channel graph (b)image segmentation

Fig.2 Quadtree algorithm renderings

\subsection{Restored image tone adjustment}

Images obtained under the fog, because of the effects of atmospheric light scattering, lead to the image of the whole partial white and pixel values are larger than true pixel value. The restored image in tone is overall darker, so tone adjustment [6] to improve the quality of the image and the restored image more in line with the requirements of the human visual. That is

$$
\begin{aligned}
& U(x)=J(x)^{\frac{d I}{d J}} e^{a I-a J \frac{d I}{d J}} \\
& T_{\text {map }}(x)=\frac{U(x)}{1+\left(\frac{1}{255}-\frac{1}{M_{U g}}\right) U_{g}(x)}
\end{aligned}
$$

The formula (10) (11), $a I ~, d I$ respectively. The mean and standard deviation of $\ln (I) ; a J$, $d J$ are the mean and standard deviation of $\ln (J) ; U_{g}(x)$ is the gray level of $U(x) ; M_{U g}$ is the maximum value of $U_{g}(x)$. From Figure 3 (a) (b) can be seen, the details of the image color adjustment after the rich, bright, visual effect can be greatly improved.

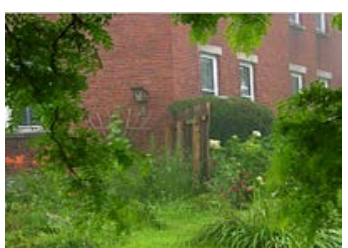

(a) Recovery image

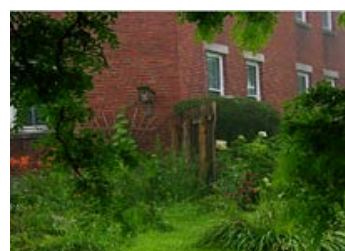

(b) Adjusted image

Fig.3 Brightness adjustment

\section{Experimental results and quality assessment}

In order to verify the feasibility and effectiveness of the algorithm, the He algorithm and the Tarel algorithm with better effect of removing fog are compared. The following experiments are simulated in the operating system Windows 7, the processor clocked at $3.10 \mathrm{GHz}$, the system memory is $4 \mathrm{~GB}$, on the use of Matlab 2013 software platform. The parameters involved in this thesis: adjusting the parameter $e=0.2$, compensation limit $\phi=0.9$, to the fog intensity coefficient $\omega=0.95$, four binary tree subdivision threshold $x=20$.

\subsection{Subjective visual effect comparison}

Based on the above parameters simulation, figure 4 shows the four groups under different scenarios for the different algorithms to fog simulation experimental results. By comparison, it is found that dark channel prior to image restoration by He et al [1] and defogging relatively clean, strong sense of hierarchy, but for local area there are still residual presence of fog, the loss of detail information, resulting in blurred vision sense. Tarel[2] due to the variations of the median filter to obtain the atmospheric optical dissipation function, the local atmospheric dissipation function estimation is inaccurate, and image restoration is still covered with a layer of mist, color distortion is serious, local regional spots exist, and the restoration effect is poor. From the first to the fourth group comparison it can be seen that this algorithm can fog clean with strong sense of hierarchy and vivid color, and has a certain visual advantage. 

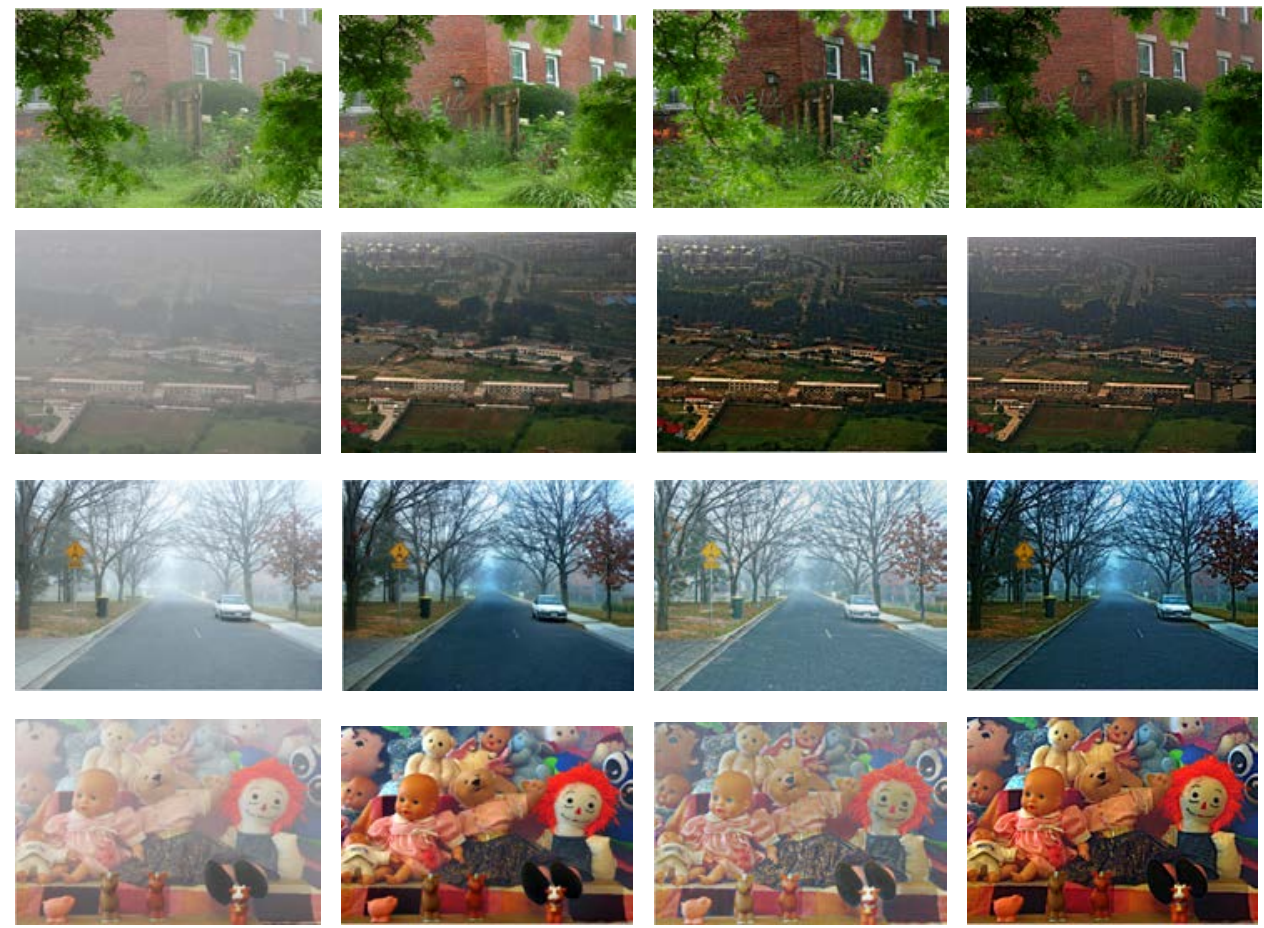

(a) fog image (b) algorithm of He (c) Tarel algorithm (d) algorithm in this paper Fig.4 Comparison of simulation results

\subsection{Quantitative assessment of fog removal}

Objective evaluation of image using Hautiere et al. Proposed the visible edge gradient method [6]. This method used the restoration of the new visible edges than, the visible edges of the standardized mean gradient and time to evaluate image restoration effect.

$$
\begin{gathered}
e=\frac{n_{r}-n_{0}}{n_{0}} \\
r=\frac{\overline{g_{r}}}{\overline{g_{0}}}
\end{gathered}
$$

Among them, $n_{0}$ and $n_{r}$ represent the number of visible edges in the original image and the image of the fog, respectively. $\overline{g_{r}}$ and $\overline{g_{0}}$, respectively, are the average gradient of the original image and the image to the fog. Usually $e$ and $r$, the greater the quality of the recovery image, the higher the quality of the $t$ algorithm used, the $t$ value of the novel, the better the real time.

As can be seen from Table 1, the $r$ and $e$ parameters of the algorithm are better than the He algorithm, and the time is much faster than the He algorithm. In this paper, the algorithm and Tarel algorithm $r$ and $e$ are not quite different, but the time is shorter. As a whole, the algorithm can improve the processing time, and it has certain advantages. 
Table.1. An objective evaluation of the simulation results

\begin{tabular}{ccccc}
\hline Image & Index & He & Tarel & This paper \\
\hline \multirow{4}{*}{ First group } & $\mathrm{e}$ & 0.109 & 0.1914 & 0.1141 \\
& $\bar{r}$ & 1.4054 & 1.8015 & 1.4751 \\
& $\mathrm{t}(\mathrm{s})$ & 11.802 & 10.757 & 1.4095 \\
Second group & $\mathrm{e}$ & 3.6727 & 3.4786 & 3.8755 \\
& $\bar{r}$ & 2.8736 & 3.0479 & 3.2283 \\
\multirow{2}{*}{ Third group } & $\mathrm{t}(\mathrm{s})$ & 9.4626 & 2.7724 & 1.3715 \\
& $\mathrm{e}$ & 0.5924 & 0.785 & 0.8129 \\
& $\bar{r}$ & 1.5142 & 2.0673 & 2.0743 \\
Fourth group & $\mathrm{t}(\mathrm{s})$ & 5.4804 & 8.7559 & 0.9919 \\
& $\mathrm{e}$ & 1.9769 & 2.0695 & 2.6386 \\
& $\bar{r}$ & 1.9911 & 2.2961 & 2.5245 \\
& $\mathrm{t}(\mathrm{s})$ & 11.685 & 4.3896 & 1.4094 \\
\hline
\end{tabular}

\section{Conclusion}

This paper presents a fast single image dehazing algorithm. Atmospheric dissipation function uses the form of transformation of mean filter to estimate, effectively avoiding the edge residual mist and partial dark phenomenon. Then use quadtree segmentation method to quickly and accurately estimate atmospheric light intensity A value reducing the time complexity, while avoiding the fog of the highlighted objects on the A value estimate of. Finally, the results of rehabilitation adjust the brightness, color get richer, clearer detail defogging renderings. Numbers of experimental results show that the algorithm ensures the fog effect, improves the speed of operation to meet the needs of real-time applications and technology and has a good practical value.

\section{Acknowledgements}

The work is supported by the National Natural Science Foundation of Shaanxi Province (Grant No. 2015JQ6221),Scientific and Technological Research Project of Science and Technology Department of Shaanxi Province (Grant No. 2012K06-16) and Special project of Education Department of Shaanxi Province (Grant No. 12JK0528).

\section{References}

[1] He K, Sun J, Tang X. Single Image Haze Removal Using Dark Channel Prior.[C]// 2013 IEEE Conference on Computer Vision and Pattern Recognition. IEEE, 2010:2341-2353.

[2] Tarel J P, Hautiere N. Fast visibility restoration from a single color or gray level image[C]// Computer Vision, 2009 IEEE 12th International Conference on. IEEE, 2009:2201-2208.

[3] Yu J, Xiao C, Li D. Physics-based Fast Single Image Fog Removal[J]. Acta Automatica Sinica, 2011, 37(2):1048-1052.

[4] Narasimhan S G, Nayar S K. Contrast restoration of weather degraded images[J]. Pattern Analysis \& Machine Intelligence IEEE Transactions on, 2003, 25(6):713-724.

[5] Kim J H, Jang W D, Sim J Y, et al. Optimized contrast enhancement for real-time image and video dehazing[J]. Journal of Visual Communication \& Image Representation, 2013, 24(3):410-425.

[6] Hautière N, Tarel J P, Aubert D, et al. Blind Contrast Enhancement Assessment by Gradient Ratioing at Visible Edges[J]. Image Analysis \& Stereology, 2008, 27(2):87-95. 